\title{
Dossier thématique : maladie de Verneuil. Conclusion
}

\section{Thematic File: Verneuil's Disease. Conclusion}

\section{A. Senéjoux \\ (C) Lavoisier SAS 2016}

La maladie de Verneuil n'est pas rare, elle est surtout mal connue d'un bon nombre de praticiens. Le coloproctologue $\mathrm{y}$ est fréquemment confronté puisque cette affection représente une étiologie fréquente des suppurations anopérinéales.

Le traitement de référence est la chirurgie, en phase aiguë pour soulager les abcès, et à la phase chronique pour supprimer les territoires cutanés touchés par la maladie, ce qui peut imposer des sacrifices cutanés importants et fait parfois recourir à des gestes chirurgicaux de reconstruction.
Le traitement médical classique repose sur les antibiotiques. De nouvelles approches sont développées par certaines équipes, fondées sur des antibiothérapies lourdes et prolongées par voie intraveineuse.

L'adalimumab a montré son efficacité pour soulager la maladie. Ce traitement a obtenu en juillet 2015 une autorisation de mise sur le marché pour traiter les formes actives modérées à sévères de la maladie, en cas d'échec du traitement systémique conventionnel. Il n'est malheureusement pour l'instant pas encore remboursé par les caisses dans cette indication.

\footnotetext{
A. Senéjoux $(\bowtie)$

6, boulevard de la Boutière, F-35760 Saint Grégoire

e-mail : agnes.senejoux@gmail.com
} 